\title{
Urgences
}

\section{Poésie et récit dans Agonie de Jacques Brault : l'intertexte mironien}

\section{Robert Dion}

Numéro 28, mai 1990

Le roman comme poétique

URI : https://id.erudit.org/iderudit/025589ar

DOI : https://doi.org/10.7202/025589ar

Aller au sommaire du numéro

Éditeur(s)

Urgences

ISSN

0226-9554 (imprimé)

1927-3924 (numérique)

Découvrir la revue

Citer cet article

Dion, R. (1990). Poésie et récit dans Agonie de Jacques Brault : l'intertexte mironien. Urgences, (28), 56-67. https://doi.org/10.7202/025589ar d'utilisation que vous pouvez consulter en ligne.

https://apropos.erudit.org/fr/usagers/politique-dutilisation/ 


\title{
Poésie et récit dans Agonie de Jacques Brault: I'intertexte mironien
}

\author{
Robert Dion
}

L'homme agonique, et c'est bien pour cela qu'il est en agonie, n'a trouvé de passage, fragile, dérisoire à ses propres yeux, qu'en la parole souffrante et joyeuse de la poésie.

Jacques Brault, « Miron le magnifique $n$, p. 40.

D'emblée, avant même que ne se déploie le récit, Agonie ${ }^{1}$ indique son rapport à la poésie. Placé en épigraphe, le poème éponyme d'Ungaretti constitue déjà, si l'on peut dire, le programme du récit à venir. C'est le poème qui semble donner au récit sa trame narrative. Mais on sait que les rapports entre les deux textes sont beaucoup plus complexes qu'il n'y paraît de prime abord. En effet, le récit de Brault contient un commentaire du poème qui l'informe. Ainsi, il apparaît que chacun des deux textes en cause est le miroir de l'autre - effet architectonique répercuté dans la thématique même de ces textes.

Je n'insisterai pas, ici, sur les relations entre le récit de Brault et son intertexte apparent, le poème d'Ungaretti. J'ai déjà abordé cette question dans un article où j'ai tenté de dégager les rapports métatextuels ${ }^{2}$ entre ces deux textes ${ }^{3}$. Je tenterai plutôt d'envisager le rapport plus ou moins crypté entre le récit de Brault et son intertexte poétique caché/exhibé, le cycle de La vie agonique de Gaston Miron ${ }^{4}$. Intertexte

1 Jacques Brault, Agonie, Montréal, Boréal Express, 1985 [1984], 77 p. Dorénavant, les références à cet ouvrage seront signalées par la seule mention du folio entre parenthèses.

2 Bernard Magné, * Métatextuel et lisibilité ", Protée, Chicoutími, vol. 14, no 1-2, printemps-été 1986, p. 77-88.

3 Voir Robert Dion, Littérarité et métatexte littéraire: l'exemple d'Agonie de Jacques Brault ", Actes du colloque international sur la littérarité, à paraître aux Presses de l'Université Laval à l'automne 1990.

4 Se pose encore ici l'éternel problème des études mironiennes, à savoir l'existence de multiples versions des mêmes textes. Par commodité (et puisqu'il s'agit, en quelque sorte, de la version officielle pour le Québec), je recourrai sur- 
cache, puisque Brault ne signale à aucun moment dans son récit la présence de l'intertexte mironien, mais néanmoins exhibé, dans le titre même du récit (ce titre, Agonie, que le professeur ne commentera pas, peut-être parce que l'ensemble du récit, commentaire et fiction, en constitue l'expansion). Il est en effet difficile de ne pas lire, sous le titre du récit de Brault, celui du cycle poétique de Miron, d'autant plus que Brault s'est fait, dans son célèbre article intitulé "Miron le magnifique " 5 , le théoricien de l'« agonique".

De nombreux indices conduisent à lire Agonie à travers le prisme de $L$ a vie agonique. Il convient d'ailleurs de relever ces indices. Plus encore, il importe de se poser les questions suivantes: en quoi Agonie constitue-t-il un discours sur la poésie, et spécialement sur la poésie de Miron? Le récit de Brault est-il modelé par cette poésie sur laquelle il tient - plus ou moins directement - un discours? Ces questions me portent au cœur même de mon objet: le discours d'un genre sur un autre genre, certes, mais aussi d'un texte sur un autre texte, par ces moyens qui permettent la plus grande intimité entre les textes, le commentaire (qui restaure une certaine distance, permettant ainsi l'explicitation de l'un par l'autre texte) et les pratiques dites hypertextuelles (qui président, au contraire, à la superposition des textes).

L'agonie évoquée dans le récit de Brault et dans La vie agonique est une lutte et une angoisse, comme le veut l'étymologie du $\operatorname{mot}^{6}$. Cette étymologie est d'ailleurs narrativisée à la toute fin du récit:

Le lieu n'est que d'angoisse, une étroitesse, un resserrement d'être ${ }^{7}$. La lutte, inutile, se donne des airs d'y croire,

tout à l'édition des Presses de l'Université de Montréal (1970); à l'occasion, j'évoquerai les variantes de l'édition Maspéro de 1981. Une édition de poche de L'homme rapaillé doit bientót paraître au Québec, qui suivra de très près la splendide édition française de 1981.

5 Jacques Brault, « Miron le magnifique " [1966], Chemin faisant, Montréal, La Presse, 1975, p. 19-48.

6 Agonie: du grec agónia, en passant par le latin ecclésiastique agonia, " lutte, angoisse " (Le petit Robern). L'agonie est l'état dans lequel le malade lutte contre la mort; elle est aussi a Extréme angoisse, grande peine d'esprit. [...]. Jésus sera en agonie jusqu'à la fin du monde " (Littré). Voir aussi Brault, "Gaston Miron ", Europe, nos 478-479 (février-mars 1969), p. 152-159: * Miron entre en poésie comme en un combat sans fin. Pressentant $\infty$ qu'il écrirait plus tard, il note des 1953: "ici l'homme est agonie ". "

7 Miron: a je suis ici à rétrécir dans mes épaules / je suis là immobile et ridé de vent " ( Monologues de l'aliénation délirante "). 
58

d'espérer que la vie triomphera. Mais il est sur son banc, défait, décomposé. (p. 77)

Ce qui, en premier lieu, unit Brault et Miron, c'est une commune définition de l'agonie, de l'agonique. Signalons, d'abord, que l'ensemble du récit de Brault est un commentaire sur l'agonie. Le titre du poème d'Ungaretti ne sera pas directement explicité dans le récit, puisqu'il est mis en scène tout au long du texte (on n'a qu'à penser à l'agonie du professeur et aux agonies multiples du narrateur ${ }^{8}$ ). Ce titre est en outre "commenté", par anticipation si je puis dire, dans "Miron le magnifique *. Cet article permet en effet à Jacques Brault de livrer sa propre conception de l'agonique par le truchement des poèmes de Miron.

Comme l'écrit Brault en marge de « Miron le magnifique », l'agonique est d'abord une intonation:

Qu'il s'agisse du pays et des luttes sociales, de l'amour partagé ou déserté, de l'histoire et du monde, de la poésie elle-même, bref quel que soit le sujet du poème, son sens obvie ou caché, toujours et jusque dans les détails d'écriture, la voix de Miron se caractérise et doit s'entendre comme une angoisse cernée de courage. ${ }^{9}$

Angoisse, courage: sont convoqués, ici encore, les topoï de l'agonie. Il appert ainsi que, pour Brault, la poésie de Miron est cette voix angoissée qui veut surmonter l'angoisse - cette angoisse de naissance qui maintient l'homme, et spécialement le «Québécanthrope *, en état d'agonie. On aura une assez juste idée du pouvoir quasi magique que Brault confere à la poésie si l'on se rappelle qu'au moment où le professeur doit lire en classe le poème d'Ungaretti, le narrateur d'Agonie note ceci :

Je regardais le petit homme gris, ainsi l'appelai-je en moimême, et j'attendais, j'attendais, avec une sourde angoisse, je ne savais quoi de capital, de décisif, et de plus angoissant que mon angoisse. II lut. (p.12)

Selon Brault, l'agonique mironien désigne la médiation du langage entre ce qui paraît devoir rester irrémédiable-

8 Agonies multiples, que le narrateur confesse comme autant de morts miniatures: faillite professionnelle (p. 10), échec de l'amour (p. 18), échec de sa vie (p. 26 et 29 ), otc.

9 Jacques Brault, * Miron le magnifique ", Chemin faisant, p. 27-28. 
ment séparé: le dedans et le dehors, l'amour et le politique, l'intime et le collectif. Les poèmes de Miron apparaissent comme les procès-verbaux d'un combat sans fin entre ces domaines cloisonnés, ainsi qu'entre le texte et le non-texte ou, pour reprendre une célèbre dichotomie, entre le poème et le non-poème; c'est en cela qu'ils témoignent d'une vie en agonie. Dans les poèmes de Miron, se rencontrent les exigences contradictoires de la vie et de la poésie. C'est sans doute pour cette raison que ces poèmes sont toujours en porte-àfaux. Chez Miron, le langage n'est pas libre de prendre son envol; il est au contraire cerné par un monde à dire.

Mais c'est paradoxalement parce que la poésie "pure» lui est interdite que Miron y parvient le plus souvent. La poésie, parole de lutte et d'angoisse, est toujours provisoire, nécessairement insatisfaisante; elle doit donc être constamment réaménagée, voire réécrite. Miron s'y emploie de telle façon qu'il s'avère peut-être, contrairement à un préjugé tenace, le plus "formaliste" des poètes québécois contemporains, en tous cas celui qui a le plus eu conscience de l'« ouverture» du texte moderne. Cet aspect n'a pas échappé à Brault, qui remarque dans "Miron le magnifique" que * Cette poésie évolue curieusement; toujours en mouvement, jamais interrompue, elle se reprend, se remodule à l'infini d'un texte à l'autre [...]. Tous les textes tremblent ainsi d'une espèce d'irrésolution à se fixer $\% .{ }^{10} \mathrm{Ce}$ sens tremblé, cette dépossession du sens, définissent en propre l'agonique et son corollaire, l'agonie.

Le sens dérobé, le sens de la poésie en l'occurrence, est d'abord à saisir par le commentaire. Chez Miron, la poésie prend parfois l'aspect d'une poésie didactique ${ }^{11}$. De même,

10 lbid., p. 23-24.

11 Le titre Recours didactique désigne dans l'édition de 1970 un poème du cycle de La vie agonique et une section complète du recueil constituée de textes en prose - ce qui établit de singulières correspondances entre écriture de la prose et ecriture de la poesie. En outre, dans les poemes mêmes, il arrive qu'on rencontre des vers aux intentions didactiques évidentes qui prennent la forme d'une prose rythmé; il en est ainsi des vers : « voici ma vraie vie - dressé comme un hangar - / debarras de l'Histoire - je la revendique / je refuse un salut personnel et transfuge / je m'identifie depuis ma condition d'humilie / je le jure sur l'obscure respiration commune / je veux que les hommes sachent que nous savons " ( "Monologues de l'aliénation délirante "). Notons au passage que deux poèmes de l'édition Maspéro ont pour sous-titre " Recours didactique " (il s'agit des a Années de déréliction" et de a Sur la place publique ") et que la section éponyme du recueil disparait. 
60

chez Brault, le roman s'assimile ponctuellement à un commentaire, à une analyse. Ainsi, dans Agonie, l'auteur multiplie les traits discursifs empruntés au discours analytique: définitions de termes relevées dans les dictionnaires (p. 12, 18, 19, 27, etc.), discours métatextuel de type grammatical ou logique (p. 13, 48-49, etc.), discours interprétatif, etc. Le commentaire tient le poème d'Ungaretti à distance et en fait l'objet de son discours ${ }^{12}$. Modalité particulière de l'agonie - qui consiste, grosso modo, en la fragmentation et en l'aliénation du moi (voir le poème intitulé L'homme rapaillé: «J'ai.fait de plus loin que moi un voyage abracadabrant / il y a longtemps que je ne m'étais pas revu» ) -, l'agonique est lisible dans la trame du récit de Brault.

La hantise de l'échec, qui selon Brault caractérise l'agonique mironien, trouve son expression dans une tension entre la nécessité de lancer l'écriture à l'assaut du sens et la peur d'échouer lamentablement ${ }^{13}$ (ce qui conduit à sans cesse recommencer le poème). Cette hantise se retrouve dans Agonie. Afin de justifier son intérêt quasi maladif pour le personnage du professeur, le narrateur du récit va jusqu'à confesser:

J'ai su à l'évidence que je n'avais pas cessé de vouloir lui dérober son secret. Pour voir en face mon propre secret. Estce bien le mot qui convient? Ma hantise plutôt, \& ma hantise » serait plus juste. La peur panique de rater définitivement, de rater quoi? (p. 11)

Ce passage illustre bien l'un des aspects dominants de l'agonique. Le narrateur fasciné, qui, une nuit durant, ne peut se déprendre de la fascination qu'exerce sur lui un personnage somme toute assez falot ${ }^{14}$, ne donne-t-il pas l'exemple de cette absence à soi qui est le propre de l'homme en agonie ${ }^{15}$ ? Quelle est donc l'identité de ce narrateur qui ne

12 Sur ces questions, je me permets encore une fois de renvoyer à mon article à paraitre (voir note 3).

13 « Partout l'agonie, ecrit Brault, et au milieu la parole qui s'essaye à une impossible médiation. " Jacques Brault, " Miron le magnifique ", Chemin faisant, p. 37.

14 Miron: « Mais je ne peux me déprendre du conglomérat / je suis le rougegorge de la forge / le mégot de survie, l'homme agonique " ( L'homme agonique ").

15 Pour que cesse l'agonie, il faut, comme le rappelle Miron, passer à travers les a tunnels de son absence" ("Héritage de la tristesse ") et retrouver sa «nue propriété" ( " L'homme agonique"). 
peut se voir vraiment que dans le miroir de l'autre (et ne peut que sombrer avec lui, comme l'alouette altérée)? Le narrateur constitue sans doute une figure exemplaire du dépaysement lequel n'est peut-être qu'une forme collective d'agonie.

Le dépaysement prend une très grande importance dans le récit de Brault (et, bien sûr, dans la poésie de Miron). Il donne le fin mot du récit et représente une image privilégiée de l'agonie: "Il n'y a pas, il n'y a jamais eu, il n'y aura jamais de pays» (p. 77), avait dit le professeur. Pendant tout le récit, le narrateur tente de se ressouvenir de cette phrase; le récit s'achève lorsqu'elle revient à sa conscience. Dans la béance de cet oubli, tient la totalité du récit. Ce dépaysement, après avoir produit sur l'étudiant un effet somatique (l'envie de vomir des premières pages), s'impose au narrateur comme une agonie, alors que plusieurs années plus tard lui et le professeur arrivent enfin au bout de leur concrète agonie (la nuit de veille de l'ex-étudiant, la mort du professeur sur son banc). L'individuel débouche ici sur le collectif: à l'agonie de l'ex-étudiant, se superpose celle du professeur, puis celle du pays. On peut donner plusieurs sens à cette métaphore du pays. Dans le contexte d'une lecture intertextuelle, on ne peut pas ne pas penser à ce vers en exergue à $L a$ vie agonique: «En étrange pays dans mon pays lui-même " (Aragon) ${ }^{16}$, ou à ces vers de Miron:

Il est triste et pêle-mêle dans les étoiles tombées

livide, muet, nulle part et effaré, vaste fantôme

il est ce pays seul avec lui-même et neiges et rocs

un pays que jamais ne rejoint le soleil natal

( * Héritage de la tristesse $*$ )

Centrale chez Miron, la thématique du pays apparaît inopinément dans le récit de Brault, à la toute dernière page. Tout se passe comme si elle n'apparaissait que pour accentuer le parallélisme avec l'œuvre de Miron, c'est-à-dire entre l'agonique et l'agonie. Mais, ce parallélisme est subtilement miné; dans Agonie, il n'est pas question d'un pays à reconquérir ou à faire, comme chez Miron:

je n'ai jamais voyagé

vers autre pays que toi mon pays

( $\propto$ Pour mon rapatriement $»$ )

16 Dans l'édition Maspéro, ce vers d'Aragon est remplacé par celui de Villon qui lui a servi de modele: " En mon pays suis en terre lointaine ". Substitution exemplaire, eu égard à la problématique de l'intertextualité. 
nous te ferons, Terre de Québec

lit des résurrections

( L'octobre $»)$

mais d'un pays problématique, impossible: d'un pays-mirage. Le récit de Brault se trouve en fait à subvertir le discours de la poésie mironienne et à mettre au jour le surcodage qui est, en 1984, celui de la " poésie du pays * des années cinquante et soixante ${ }^{17}$. Cette prise de distance vis-à-vis de l'intertexte est cependant respectueuse, et le dialogisme ne prend pas ici la forme d'une carnavalisation parodique.

Par ailleurs, il est tentant d'établir une analogie entre le personnage du professeur dans Agonie et le "protagoniste " des poèmes de La vie agonique ${ }^{18}$. En effet, l'« homme gris * du récit de Brault n'est pas sans rappeler le "damned Canuck", l'«homme du cheap way mironien. C'est la même figure du dépossédé "silencieux raboteux rabot[é]*, la même aliénation et une pareille insignifiance. Le professeur, comme le je des poèmes, "est dans l'échec par-dessus la tête" 19 . On aura un meilleur portrait de la condition de ce sous-homme, le Québécois aliéné, à lire la correspondance de Gaston Miron à Claude Haeffely publiée récemment; qu'il suffise d'en parcourir les extraits suivants:

Ma poésie n'est pas s'en [sic] se ressentir de ce climat. C'est l'angoisse de coux qui ne veulent pas disparaître et qui rejoint l'angoisse de l'homme tout court atteint dans sa liberté la plus personnelle. 20

J'écris dans l'impossible. Je rate. Je bafouille tous les alphabets. Je me cherche les indices de moi. Je m'affaisse. Perdant pied et lucidité. 21

Tout simplement et consciemment, je me suis aliéné à moimème. Aliénation de toute affectivité, sans pour autant

17 Voir à ce sujet l'article de Laurent Jenny, a La stratégie de la forme ", Poétique, Paris, $n^{\circ} 27,1976$, p. 257-281.

18 Je signale qu'il y a aussi une analogie entre le personnage du professeur et l'énonciateur de l'article intitulé a Une grammaire du cosur " (c'est-à-dire Brault lui-même), qui raconte l'histoire d'un amour d'enfance exactement similaire à celui qu'évoque le professeur dans Agonie. Voir Jacques Brault, a Une grammaire du cceur $n$, Chemin faisant, p. 17-18.

19 Gaston Miron, $\dot{A}$ bout portant, Correspondance de Gaston Miron a Claude Haeffely, Montréal, Leméac, 1989, p. 48.

20 Ibid., p. 22.

21 Ibid., p. 41. 
démissionner sur le plan de l'action. Aliénation aussi sur le plan de la création par voie de conséquence, puisque celle-ci était liée à mon affectivité. C'est tout. ${ }^{22}$

$\mathrm{Au}$ total, le récit de Brault apparaît comme un * travail de transformation et d'assimilation de plusieurs textes ${ }^{23}$. Il évoque et transforme quelques poèmes du corpus mironien avec lesquels il entre en résonance thématique ${ }^{24}$. Il fait cependant plus qu'entrer en résonance thématique avec ce corpus. C'est là où je veux maintenant en venir.

Commentaire du poème d'Ungaretti, Agonie constitue, par le fait même, le commentaire d'un roman sur la poésie, d'un genre sur un autre genre. Le roman vient, en quelque sorte, meubler les interstices du poème. D'un poème qui, paradoxalement, "progresse" en se recommençant trois fois avec trois actants différents (l'alouette, la caille, le chardonneret), il fait un récit plus linéaire où deux actants (le narrateur et le professeur), par un jeu de miroir, finissent par n'en faire qu'un seul. Le récit illustre concrètement l'ainterpénétration" des deux genres, le poème d'Ungaretti réapparaissant dans les titres des chapitres, dans le commentaire luimême (qui souvent le paraphrase) et dans la trame narrative du récit. Mais, il y a autre chose. Évocation plus ou moins directe de La vie agonique, le récit de Brault peut passer pour sa réecriture. On entre ici dans le domaine de l'intertextualité stricto sensu, et plus précisément de l'hypertextualité. J'avance l'hypothèse - sans doute téméraire - que le récit de Brault reprend sur le mode romanesque le poème intitulé *Héritage de la tristesse ${ }^{25}$. La superposition des deux textes me paraît d'abord justifiée par leur clausule respective:

22 lbid., p. 131.

23 Jenny, op. cit., p. 262.

24 Le récit entre également en résonance thématique avec d'autres textes de Brault. Je ne citerai qu'un exemple extrait de Moments fragiles, recueil de poèmes publié la méme année qu'Agonie: \& La cheveche aux grands yeux d'enfant / s'étonne tout au bout du monde / voir la vacuité de ce printemps / elle ne craint pas de faiblir mourir / elle craint seulement de survivre / à cette agonie". Je note en vrac quelques analogies avec Agonie: la métaphore de l'oiseau (la cheveche est un rapace), l'utilisation des verbes à l'infinitif, l'allusion à l'agonie. Jacques Brault, Moments fragiles, Saint-Lambert, Norolt, 1984, p. 89.

25 Rappelons que ce poème, le second de La vie agonique dans l'édition des Presses de l'Université de Montréal, en était à l'origine le poème d'ouverture (voir Liberté, Montréal, vol. 5, n 3, mai-juin 1963). Il avait alors pour titre 
64

les vents qui changez les sorts de place la nuit vents de rendez-vous, vents aux prunelles solaires vents telluriques, vents de l'âme, vents universels vents ameutez-vous, et de vos bras de fleuve ensemble enserrez son visage de peuple abîmé, redonnez-lui la chaleur

et la profuse lumière des sillages d'hirondelles (" Héritage de la tristesse »)

Alouette, caille, chardonneret, quand vous reviendrez du soleil, quand vous rentrerez au pays agonisant, vos ombres se déchiquetant aux aspérités du sol, chantez, je vous prie, chantez à vous étouffer. (Agonie, p. 77)

Ces deux passages ont en commun un ton relativement rare: celui de l'exhortation. Le poème comme le roman se terminent par une incitation à faire quelque chose, manifestée par une série de verbes à l'impératif: « armeutez *, « enserrez " et "redonnez » chez Miron, «chantez " (deux fois) chez Brault. De plus, les deux textes se signalent par une même syntaxe anaphorique. Celle-ci, évidente chez Miron, modèle également le texte de Brault, qu'on pourrait sans peine redisposer ainsi:

Alouette, caille, chardonneret,

quand vous reviendrez du soleil, quand vous rentrerez au pays agonisant,

vos ombres se déchiquetant aux aspérités du sol, chantez, je vous prie, chantez à vous étouffer.

Les parentés lexicales entre les deux clausules sont tout aussi frappantes: au "soleil» du récit, correspondent les "prunelles solaires " et la "profuse lumière " du poème; les " hirondelles " (au pluriel) du poème répondent à l'« alouette», à la «caille» et au "chardonneret » du récit; et au " peuple abîmé » de Miron, renvoie le « pays agonisant" de Brault.

Toutefois, par delà ces correspondances, s'opère entre le poème de Miron et le récit de Brault un véritable renversement sémantique, comparable à celui que j'ai noté plus haut à propos de la notion de pays (qui, de pays à faire chez Miron, devient

a Tristesse, 8 ma pitie, mon pays , titre fort éloquent en regard du récit de Brault publié 21 années plus tard, qui se termine précisément, après moult développements sur la tristesse et la pitié, par l'évocation du pays. 
pays impossible chez Brault). Dans le poème, les * vents telluriques » aux " bras de fleuve *, qui renvoient autant aux domaines aquatique et terrestre que céleste, annoncent les paisibles et aériens sillages d'hirondelles, tandis que l'alouette, la caille et le chardonneret du récit, qui ressortissent, eux, à l'élément aérien, paraissent irrémédiablement promis à la chute (par le truchement de leurs "ombres") et à l'étouffement. Dans un cas, les vents terribles mais bénéfiques rendent au peuple abîmé la "chaleur " et la « lumière (par le biais des «sillages d'hirondelles»). Dans l'autre, les oiseaux, en rentrant au pays agonisant, sont attirés au sol qui les déchiquette (métonymiquement: il déchiquette les "ombres") et ne chantent que pour mieux s'étouffer. À la conclusion relativement optimiste de Miron, s'oppose donc, dans une parfaite antisymétrie, la clausule désespérée du récit de Brault.

Ce n'est pas que dans les clausules que ces deux textes se font mutuellement écho. Entre eux, se tisse tout un réseau de résonances qui me porte à croire que l'un a pu servir, sinon de synopsis, du moins de point de départ (d'hypotexte) à l'autre. Qu'est-ce que le récit de Brault, en effet, sinon celui d'un «Héritage de la tristesse "? (Une tristesse, en l'occurrence, transmise de mère en fils.) Je ne m'engagerai pas ici dans la voie des vagues analogies et des échos métaphoriques; je voudrais seulement signaler qu'on trouve dans chacune des cinq strophes du poème de Miron des syntagmes qui ont pu servir de matrice au récit de Brault.

Par exemple, on relève, dans la première strophe, les vers que je citais plus haut ( "Il est triste et pêle-mêle dans les étoiles tombées / livide, muet, nulle part et effaré, vaste fantôme / il est ce pays seul [...]\% / etc.), qui renvoient au Il n'y a pas, il n'y a jamais eu, il n'y aura jamais de pays de la fin d'Agonie. On remarque aussi dans cette strophe deux métaphores (presque des synesthésies) qui présentent une étonnante parenté avec les «alouettes altérées* du poème d'Ungaretti: Miron y évoque "un sommeil désaltérant/pareil à l'eau dans la soif vacante des graviers » (v. 5-6); cette " soif vacante " est sans doute à rapprocher de l'explication que donne le professeur au sujet du curieux *altérées " du poème d'Ungaretti: "La soif creuse un besoin, un désir de change. ment." (p. 14) - une vacance? La deuxième strophe, pour sa part, pourrait évoquer l'épisode du récit où l'on voit le professeur gisant sur son banc, le il du poème étant susceptible, 
par son ambiguïté même, de désigner à la fois le «pays " (ce qu'il désigne effectivement dans le poème de Miron) et un sujet humain; quant au je qui inaugure la strophe, il fait figure de témoin apercevant le $i l$ brûlant « en longs peupliers d'années et d'oubli * (v. 10). Cette confusion du pays et de l'humain dans un $i l$ ambigu, sans antécédent immédiat (le « pays * désigné par ce il n'est mentionné qu'au quatrième vers, dans la première strophe), est tout à fait dans l'esprit du récit de Brault. Les troisième et quatrième strophes, enfin, où règne toujours la confusion entre un pays *humanisé " et un sujet humain, peuvent être assimilées à une description du professeur, principal protagoniste d'Agonie: ce $i l$ *prostré " (v. 13) "parmi ses haillons de silence aux iris de mourant» (v. 15), arborant un * sourire échoué du pauvre avenir avili» (v. 16) ne peut-il pas désigner, outre le "pays que jamais ne rejoint le soleil natal " (v. 4), le gris et falot professeur de beauté ? Et ces vers:

démuni, il ne connaît qu'un espoir de terrain vague qu'un froid de jonc parlant avec le froid de ses os le malaise de la rouille, l'à-vif, les nerts, le nu dans son large dos pâle les coups de couteaux cuits il vous regarde, exploité, du fond de ses carrières

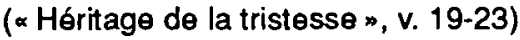

en plus de dépeindre un pays aliéné, ne peignent-ils pas aussi le médiocre professeur? n'annoncent-ils pas l'« espoir de terrain vague * que fait naître l'épisode de l'excursion à Rivière-des-Prairies (où le futur professeur croit retrouver, après une errance dans un no man's land désert et glacé, la petite Michèle de sa prime enfance)? Et comment ne pas attribuer aux étudiants les «coups de couteaux* dans le elarge dos pâle" (gris?) du professeur, lequel finira bien d'ailleurs par tourner vers eux son regard exténué ? Quant à la dernière strophe du poème, qui constitue sa clausule, j'ai suffisamment insisté sur ses parallélismes formels et thématiques avec Agonie pour qu'il soit nécessaire d'y revenir ici.

Le rapport entre Agonie et La vie agonique est d'abord tet avant tout, on l'a vu, un rapport d'intertextualité, dans la mesure où le récit de Brault réactive certains éléments déjà contenus dans les poèmes de Miron, en plus de réactiver certaines idées sur la poésie de Miron déjà exprimées dans l'essai intitulé « Miron le magnifique*. En second lieu, on peut affirmer que le récit et le cycle poétique sont également liés par des rapports d'intergénéricité; de même qu'Agonie 
constitue un commentaire du poème d'Ungaretti, il s'avère un commentaire du cycle poétique de Miron - tout comme l'agonie braultienne représente un avatar de l'agonique mironien. Il appert ainsi que dans la fiction au même titre que dans le commentaire, Agonie est un discours sur la poésie, sur celle d'Ungaretti comme sur celle de Miron. Dans ce récit, est affirmée entre autres la valeur didactique de la poésie, ne serait-ce que parce que le poème d'Ungaretti sert de texte de référence dans le cours de philosophie du professeur-protagoniste. À cette valeur didactique de la poésie s'ajoute la valeur didactique du roman, dans la mesure où Agonie prend ouvertement le parti d'analyser un texte poétique - procédé anti-romanesque s'il en est, du moins $a$ priori. Enfin, si je considère le récit de Brault comme le point de jonction entre son discours et la poésie mironienne, force m'est de constater qu'y interferent deux didactiques complémentaires, tout comme s'y rencontrent deux genres littéraires.

C'est sans doute le didactisme du récit braultien qui lui permet d'assimiler en profondeur, dans sa trame même, celui de la poésie de Miron. L'une des caractéristiques fondamentales de cette intertextualité réside dans le fait qu'Agonie et La vie agonique sont, entre autres, des textes didactiques ce qui, en l'occurrence, rend le texte de Miron particulièrement apte à être saisi par un autre texte qui en est le commentaire indirect et la récriture hypertextuelle. Il s'agit évidemment, dans le récit de Brault et dans le cycle poétique de Miron, de deux didactiques différentes. Le premier est un métadiscours analytique; le second, un discours interprétatif à caractère souvent prescriptif. Il reste, néanmoins, que c'est la rencontre de deux intentés proprement didactiques qui donne à cette cohabitation des textes son caractère propre; c'est cette rencontre qui rend si précieuse celle que Brault a faite, un jour, des textes de Miron. 